\title{
Studies of multi-quasiparticle K-isomers in rare-earth and trans-fermium nuclei
}

\author{
F.G. Kondev 1,a , G.D. Dracoulis², T.L. Khoo ${ }^{3}$, G.J. Lane ${ }^{2}$, A.P. Byrne' ${ }^{2}$, T. Kibédi², I. Ahmad ${ }^{3}$, B. Back ${ }^{3}$, M.P. Carpenter ${ }^{3}$, \\ R.V.F. Janssens ${ }^{3}$, T. Lauritsen ${ }^{3}$, C.J. Lister ${ }^{3}$, D. Peterson ${ }^{3}$, D. Seweryniak ${ }^{3}$, S. Zhu ${ }^{1,3}$, P. Chowdhury ${ }^{4}$, and S.K. Tandel ${ }^{4}$ \\ 1 Nuclear Engineering Division, Argonne National Laboratory, Argonne, IL 60439, USA \\ 2 Department of Nuclear Physics, R.S.Phys.S.E, Australian National University, Canberra, ACT 0200, Australia \\ 3 Physics Division, Argonne National Laboratory, Argonne, IL 60439, USA \\ 4 Department of Physics, University of Massachusetts Lowell, Lowell, MA 01854, USA
}

\begin{abstract}
Nuclear K-isomers play an important role in understanding the structure of deformed axially symmetric nuclei. Examples are presented of recent studies in the rare-earth region (A 180) using deep-inelastic and multinucleon transfer reactions, and in the trans-fermium region (A 250) using fusion-evaporation reactions. A specific two-level mixing scenario is invoked to explain the unusual decay of the $\mathrm{K}^{\pi}=13^{+}$isomer in ${ }^{174} \mathrm{Lu}$. The identification of 2- and 4-quasiparticle isomers in ${ }^{254}$ No is discussed and predictions of similar isomers in neighboring No and $\mathrm{Rf}$ nuclei are presented.
\end{abstract}

\section{Introduction}

Gamma-ray spectroscopy studies of deformed rare-earth (A 180) and trans-fermium (A 250) nuclei provide valuable information on the properties of high-K isomers and on the seniority dependence of the major residual interactions in deformed nuclei. Most of the studies in the A 180 region so far were focused on neutron-deficient nuclei that are accessible by heavy-ion fusion evaporation reactions. Recently, deepinelastic and multi-nucleon transfer reactions, in conjunction with time correlated $\gamma$-ray coincidence techniques and large $\gamma$-ray detector arrays, have been shown to represent a powerful tool to study nuclei near the valley of stability, where most of the long-lived isomers occur, as well as to access neutron-rich nuclei [1-8]. In addition, the discovery and characterization of high- $\mathrm{K}$ isomeric states in the trans-fermium region play an important role in better understanding the structure of the heaviest elements and in testing theoretical predictions of their stability.

\section{Recent studies in the rare-earth region}

A series of time-correlated, $\gamma$-ray coincidence measurements were carried out at the ATLAS facility, Argonne National Laboratory (ANL), using $820-\mathrm{MeV}^{136} \mathrm{Xe}$ pulsed beams (1 ns on/825 ns off) incident on targets of ${ }^{174} \mathrm{Yb},{ }^{176} \mathrm{Yb},{ }^{175} \mathrm{Lu}$, ${ }^{176} \mathrm{Lu}$ and a number of heavier isotopes. In a fewf cases where longer-lived isomers were present, $10 \mu \mathrm{s} / 30 \mu \mathrm{s}$ and $1 \mathrm{~ms} / 3 \mathrm{~ms}$ beam pulsing regimes were also used, but events were collected only between the beam bursts. The beam conditions ( $\sim 20 \%$ above the Coulomb barrier) allowed transfers of nucleons between the target and projectile, and, hence, resulted in population of nuclei near the valley of stability, or on the neutron-rich side, that were not accessible previously. The $\gamma$-rays from the reaction products were detected by the

a Presenting author, e-mail: kondev@anl.gov

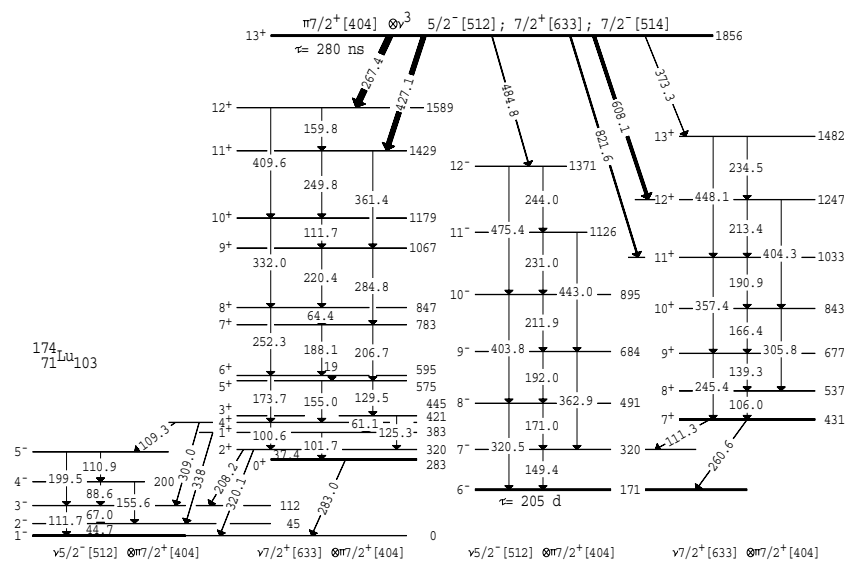

Fig. 1. Partial level scheme of ${ }^{174} \mathrm{Lu}[8]$.

Gammasphere spectrometer, comprised for these experiments of 100 Compton-suppressed Ge detectors. Many new high-K isomers were discovered in the A 180 region and their structures were characterized [4-8].

An example of a case where traditional reactions cannot access high-spin states is the odd-odd nucleus ${ }^{174} \mathrm{Lu}(Z=$ 71 and $\mathrm{N}=103$ ). It has been studied previously using $\left({ }^{7} \mathrm{Li}, 3 \mathrm{n}\right)$ and $(\mathrm{p}, 3 \mathrm{n})$ reactions (see for example ref. [9] and references therein). Intrinsic structures and corresponding rotational bands associated with the $\mathrm{K}^{\pi}=1^{-}$ground state and the $6^{-}$isomer $\left(\mathrm{T}_{1 / 2}=142(2) d\right.$ [9]) originating from the antiparallel and parallel couplings of the $\pi 7 / 2^{+}[404]$ and $v 5 / 2^{-}$[512] Nilsson orbitals, respectively, as well as those associated with the $\pi 7 / 2^{+}[404] \otimes v 7 / 2^{+}[633]$ configuration $\left(\mathrm{K}^{\pi}=0^{+}\right.$and $\left.7^{+}\right)$were observed. We have extended the level scheme of this nucleus up to a spin of $26 \hbar$ and an excitation energy of $6 \mathrm{MeV}$. The partial level scheme of ${ }^{174} \mathrm{Lu}$ is shown in figure 1, where the multiple decays of the newly-identified $\mathrm{K}^{\pi}=13^{+}$isomer $\left(\mathrm{T}_{1 / 2}=194(17) \mathrm{ns}\right)$ are presented [8]. A $\gamma$-ray spectrum in the out-of-beam region produced by gating on transitions within the $\mathrm{K}^{\pi}=0^{+}$and $6^{-}$bands is given in 


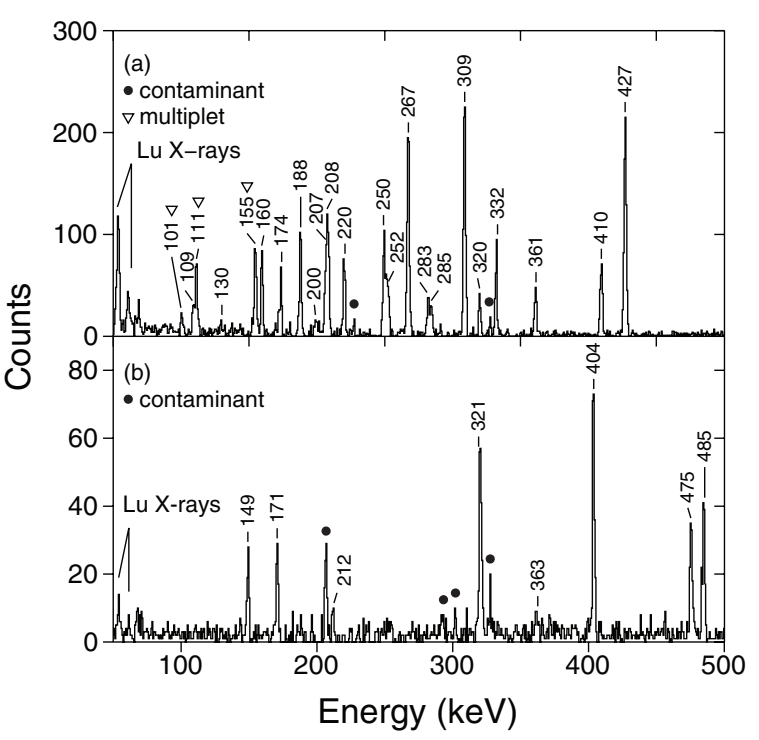

Fig. 2. Double gated gamma-ray coincidence spectra in the out-ofbeam region; (a) summed $220 / 250 \mathrm{keV}$ and $155 / 188 \mathrm{keV}$ gates in the $\mathrm{K}^{\pi}=0^{+}$band; (b) summed $404 / 475 \mathrm{keV}, 321 / 485 \mathrm{keV}$ and $475 / 485 \mathrm{keV}$ gates in the $\mathrm{K}^{\pi}=6^{-}$band.

figure 2. In addition to the $\mathrm{K}^{\pi}=13^{+}$isomer, a 4-quasiparticle isomer with $\mathrm{K}^{\pi}=14^{-}\left(\mathrm{T}_{1 / 2}=38\right.$ (4) ns), a 6-quasiparticle isomer with $\mathrm{K}^{\pi}=21^{+}\left(\mathrm{T}_{1 / 2}=97(10) \mathrm{ns}\right)$ and a 8-quasiparticle isomer with $\mathrm{K}^{\pi}=26^{-}\left(\mathrm{T}_{1 / 2}=243\right.$ (19) ns) were also discovered [10]. The configuration assignments of these states were supported by results from multi-quasiparticle calculations, similar to those presented elsewhere [5]:

$-\mathrm{K}^{\pi}=13^{+}, \pi 7 / 2^{+}[404] \otimes v^{3}\left(5 / 2^{-}[512], 7 / 2^{-}[514]\right.$, $\left.7 / 2^{+}[633]\right)$

$-\mathrm{K}^{\pi}=14^{-}, \pi 7 / 2^{+}[404] \otimes v^{3}\left(5 / 2^{-}[512], 7 / 2^{+}[633]\right.$, $\left.9 / 2^{+}[624]\right)$

$-\mathrm{K}^{\pi}=21^{+}, \pi^{3}\left(7 / 2^{-}[523], 7 / 2^{+}[404], 9 / 2^{-}[514]\right) \otimes$ $v^{3}\left(5 / 2^{-}\right.$[512], 7/2- [514], 7/2 $\left.[633]\right)$

$-\mathrm{K}^{\pi}=26^{-}, \pi^{3}\left(7 / 2^{-}[523], 7 / 2^{+}[404], 9 / 2^{-}[514]\right) \otimes$ $v^{5}\left(1 / 2^{-}[521], \quad 5 / 2^{-}[512], \quad 7 / 2^{-}[514], \quad 7 / 2^{+}[633]\right.$, $\left.9 / 2^{+}[624]\right)$.

The long-lived nature of the observed ${ }^{174} \mathrm{Lu}$ isomers in principle occurs because the depopulating transitions are $\mathrm{K}$-forbidden. For a transition of multipole order, $\lambda$, the reduced hindrance factor per degree of K-forbiddenness, $\mathrm{f}_{v}$, where $v=\delta \mathrm{K}-\lambda$, is defined as $\mathrm{f}_{v}=\mathrm{F}_{W}^{1 / v}$, where $\mathrm{F}_{W}=\mathrm{T}_{1 / 2}^{\gamma} / T_{1 / 2}^{W}$ and $\mathrm{T}_{1 / 2}^{\gamma}$, and $\mathrm{T}_{1 / 2}^{W}$ are the partial $\gamma$-ray and the Weisskopf estimate half-lives, respectively. A remarkable feature of the decay of the $\mathrm{K}^{\pi}=13^{+}$isomer is the preferential decay to the $\mathrm{I}^{\pi}=11^{+}$and $12^{+}$levels of the $\mathrm{K}^{\pi}=0^{+}$band. For example, the reduced hindrance factor obtained for the 427-keV E2 transition to the $\mathrm{I}^{\pi}=11^{+}$level is $\mathrm{f}_{v}=1.9$, a value that is surprisingly low given the fact that the transition is $\Delta \mathrm{K}=13$ forbidden. Such a fast transition cannot be explained using conventional Coriolis mixing and its occurrence is attributed to an accidental mixing between the isomer and the $\mathrm{I}^{\pi}=$ $13^{+}$member of the $\mathrm{K}^{\pi}=0^{+}$band [8]. Unfortunately, this $\mathrm{I}^{\pi}=13^{+}$level has not yet been identified and its energy has been estimated using an extrapolated value for the band member, placing it (after mixing) only 11.6 (25) $\mathrm{keV}$ above the $\mathrm{K}^{\pi}=13^{+}$isomer. By assuming a two-level interaction, the mixing amplitude, $\beta$, of the collective state into the $\mathrm{K}^{\pi}=13^{+}$ isomer can be estimated as:

$$
\beta^{2}=\alpha^{2} \times B(E 2 ; 427)^{\text {expt }} / B(E 2)^{\text {coll }}
$$

where $\mathrm{B}(\mathrm{E} 2)^{\text {coll }}=(5 / 16 \pi) \mathrm{Q}_{0}^{2}|\langle I K 20 \mid I-2 K\rangle|^{2}$ and $\mathrm{Q}_{0}=$ 7.65 (9) $e \mathrm{~b}$ (the average of the ${ }^{173} \mathrm{Lu}$ and ${ }^{175} \mathrm{Lu}$ values). The deduced value of $\beta=1.64(6) \times 10^{-3}$ implies a mixing matrix element of only $\sim 19(4) \mathrm{eV}$, a value comparable to those reported for chance mixing in the nearby nuclei ${ }^{176} \mathrm{Lu}$ [11], ${ }^{179} \mathrm{Ta}[5]$ and ${ }^{182} \mathrm{Re}$ [12], where similar abnormally-fast decays were observed. The value of the mixing matrix element in all these cases is comparable to that known for atomic scale interactions, but is orders of magnitude lower than that observed between interacting nuclear collective levels, which are typically of the order of tens of $\mathrm{keV}[13,14]$. The unusually fast decay in ${ }^{174} \mathrm{Lu}$ is, therefore, not a consequence of some erosion of the $\mathrm{K}$-quantum number, but is rather due to a chance mixing between two different, but closely spaced, nuclear quantum states. The very small interaction matrix element is an indication of the clear separation in K-configuration space of the unperturbed states.

\section{Recent studies in the trans-fermium region}

Nuclei in the trans-fermium region near $\mathrm{Z} \sim 102$ and $\mathrm{N} \sim 152$ are predicted to be well deformed with $\beta_{2} \sim 0.25$ [15] and this has been recently confirmed by observations of rotational structures in several $\operatorname{Fm}(Z=100)$ and No $(Z=102)$ isotopes [16-19]. Their single-particle spectra are dominated by high-K orbitals near both the proton and neutron Fermi surfaces [20]. These conditions favor the existence of high-K multi-quasiparticle states at relatively low excitation energy, some of which can be expected to be long-lived due to the conservation of the $\mathrm{K}$ quantum number. In addition, the deformed sub-shell gaps in the neutron and proton singleparticle spectra near $N=152$ and $Z=100$, respectively, create a robust barrier against fission and this results in an enhanced stability of these nuclei owing to the increased shell-correction energy. These gaps also isolate the important $v 7 / 2^{+}[624]$, $\nu 9 / 2^{-}[734]$ and $\pi 7 / 2^{-}[514], \pi 9 / 2^{+}[624]$ orbitals that can form favored 2-quasiparticle, $\mathrm{K}^{\pi}=8^{-}$states in the region. Since the pairing correlations are significantly reduced in these multiquasiparticle configurations, due to the effect of blocking, one may expect that the $\mathrm{K}^{\pi}=8^{-}$states gain additional stability against spontaneous fission [21].

The challenges in the spectroscopic studies of isomers in very heavy nuclei are manifold: a) low production cross sections; b) the presence of low-energy transitions resulting in the emission of conversion electrons rather than $\gamma$-rays; and c) long lifetimes leading to experimental difficulties owing to the large probability for random coincidences.

A very long-lived, 2-quasiparticle isomer in ${ }^{254}$ No was initially reported by Ghiorso et al. [22] and tentatively assigned $\mathrm{K}^{\pi}=8^{-}$, although its excitation energy and decay properties were not revealed. Recently, an unambiguous identification was made at ANL where a shorter-lived 4-quasiparticle 
isomer was also discovered [23]. Similar, but not identical, assignments were made in independent measurements performed at the Accelerator Laboratory of the University of Jyväskylä, Finland [24].

The ANL experiment was carried out at the ATLAS facility using the Fragment Mass Analyzer (FMA). Excited states of ${ }^{254}$ No were populated by the ${ }^{208} \mathrm{~Pb}\left({ }^{48} \mathrm{Ca}, 2 \mathrm{n}\right)$ reaction at $217 \mathrm{MeV}$ beam energy (in the middle of the target), with an average beam current of $50 \mathrm{pnA}$. The $\sim 0.5 \mathrm{mg} / \mathrm{cm}^{2}$ thick target was mounted on a rotating wheel and the beam was wobbled by $\sim 3 \mathrm{~mm}$ to extend the target lifetime. Evaporation residues were transported and identified by their mass to charge ratio using the FMA, then implanted into a 140 $\mu \mathrm{m}$ thick double-sided silicon strip detector (DSSD) with $40 \times 40$ pixels of $1 \mathrm{~mm} \times 1 \mathrm{~mm}$ size. Three four-fold clover Ge detectors surrounded the DSSD at the FMA focal plane to detect $\gamma$-rays following the decay of long-lived states. The identification of ${ }^{254}$ No isomers was made by the observation of the characteristic sequence of implanted recoils, one or more isomeric decays (conversion and Auger electrons) followed by the characteristic $8.1-\mathrm{MeV} \alpha$-decay within the same pixel. The proposed decay scheme from the ANL work [23] is shown in figure 3 . It reveals the presence of two isomers, one at the excitation energy of $\sim 1.3 \mathrm{MeV}$ with $\mathrm{K}^{\pi}=8^{-}$ $\left(\mathrm{T}_{1 / 2}=266(10) \mathrm{ms}\right)$ that is associated with the earlier findings by Ghiorso et al. [22], the second with $\mathrm{K}^{\pi}=\left(14^{+}\right)\left(\mathrm{T}_{1 / 2}=\right.$ $171(9) \mu \mathrm{s})$ placed above the $\mathrm{K}^{\pi}=8^{-}$isomer. Similarly, two isomers were reported by Herzberg et al. [24], although different conclusions were made about their structure, as discussed below.

Predictions of the properties of high-K states in nobelium ( $Z=102)$ and rutherfordium $(Z=104)$ nuclei have been made using multi-quasiparticle calculations. The procedure begins with a set of single-particle states obtained using the WoodsSaxon potential with the universal parametrization [25] and the equilibrium deformations of ref. [15]. The number of single-particle basis states is truncated to include only orbitals originating from the $N=4,5$ and 6 oscillator shells for the protons (64 levels) and $N=5,6$ and 7 oscillator shells for the neutrons ( 85 levels). It should be noted that the single-particle energies given by the Woods-Saxon potential accurately describe (within $0.3 \mathrm{MeV}$ ) the experimental singleparticle states derived from the observed 1-quasiparticle energies [20]. The energies of multi-quasiparticle states are calculated self-consistently by taking into account the effect of pairing using the Lipkin-Nogami prescription, with blocking and particle number conservation included, as described by Nazarewicz et al. [26]. Fixed pairing strengths of $\mathrm{G}_{\pi}=24 / A$ $\mathrm{MeV}$ and $\mathrm{G}_{v}=17.8 / A \mathrm{MeV}$ were used, which, on average, reproduce the pairing gaps in the region [15]. The energies of the 2-quasiparticle states (relative to the $0^{+}$ground "vacuum" state) were subsequently corrected by $|V|=100 \mathrm{keV}$ to account for the effect of residual spin-spin interactions. The sign of $V$ is negative for spin-singlet configurations and positive for triplet ones. For the 4-quasiparticle states, $E_{4 q p}\left(\pi^{2} v^{2}\right)=$ $E_{2 q p}\left(\pi^{2}\right)+E_{2 q p}\left(v^{2}\right)-V$, where $V=150 \mathrm{keV}$ is assumed to account for the additional effect of the proton-neutron interactions within the multi-quasiparticle configuration (see for example ref. [27] and references therein).

The predicted excitation energies for high-K states in series of No $(Z=102)$ and $\operatorname{Rf}(Z=104)$ isotopes are

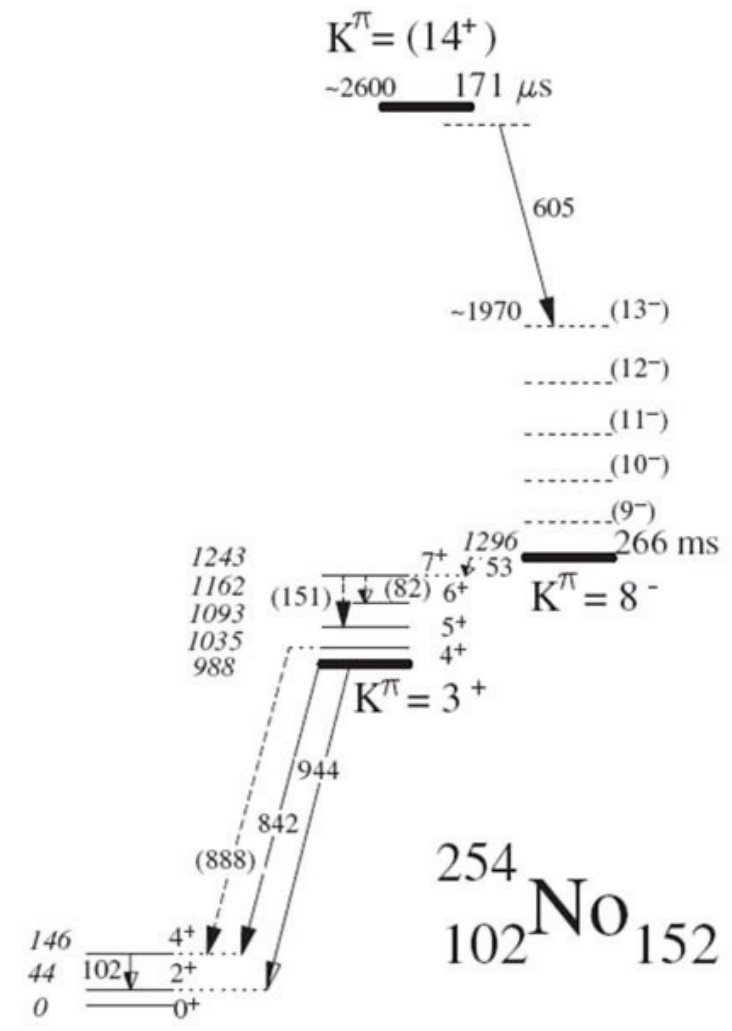

Fig. 3. Partial level scheme of ${ }^{254}$ No [23].

provided in figure 4 . In ${ }^{254}$ No $(N=152)$, the $\left(\pi^{2}\left(1 / 2^{-}[521]\right.\right.$, $\left.7 / 2^{-}[514]\right)_{3^{+}}$state is calculated at an excitation energy of 0.89 $\mathrm{MeV}$ (not shown in fig. 4) and the $\pi^{2}\left(7 / 2^{-}[514], 9 / 2^{+}[624]\right)_{8^{-}}$ state at $1.4 \mathrm{MeV}$. These two configurations are associated with the $\mathrm{K}^{\pi}=3^{+}$and $8^{-}$states observed at $0.99 \mathrm{MeV}$ and $1.3 \mathrm{MeV}$, respectively [23]. As can be seen in figure 4, the competing $v^{2}\left(7 / 2^{+}[624], 9 / 2^{-}[734]\right)_{8_{1}^{-}}$configuration is predicted at much higher energy. This is due to the fact that at $N=152$ both the $v 7 / 2^{+}[624]$ and $v 9 / 2^{-}[734]$ orbitals are located below the neutron Fermi surface and because of the sizable gap in the neutron single-particle spectrum at $\beta_{2} \sim 0.25$ and $N=152$. Our conclusions differ from those presented in ref. [24], where the $v^{2}\left(7 / 2^{+}[624], 9 / 2^{-}[734]\right)_{8_{1}^{-}}$configuration is proposed to be favored at 1.27 MeV. Studies of Xu et al. [28] and Soloviev [29] predicted the alternative $v^{2}\left(7 / 2^{+}[613], 9 / 2^{-}[734]\right)_{8^{-}}$configuration at lower energy (labeled $8_{2}^{-}$in fig. 4). Since the $7 / 2^{+}[613]$ orbital is located above the $N=152$ sub-shell gap, such a placement would require that the size of this gap be significantly reduced in ref. [28,29]. This contradicts experimental observations in the region and predictions made by other authors (see for example ref. [30] and references therein). There is a significant difference between our work and ref. [24] in the interpretation of the structure of the 4-quasiparticle isomer in ${ }^{254}$ No. While our calculations clearly isolate the $\pi^{2}\left(7 / 2^{-}[514]\right.$, $\left.9 / 2^{+}[624]\right)_{8^{-}} \otimes v^{2}\left(3 / 2^{+}[622], 9 / 2^{-}[734]\right)_{6^{-}}$configuration and hence $\mathrm{K}^{\pi}=14^{+}$, the work of Herzberg et al. [24] proposes $\mathrm{K}^{\pi}=16^{+}$and the $\pi^{2}\left(7 / 2^{-}[514], 9 / 2^{+}[624]\right)_{8^{-}} \otimes v^{2}\left(7 / 2^{+}[624]\right.$, $\left.9 / 2^{-}[734]\right)_{8^{-}}$configuration (labeled $16_{1}^{+}$in fig. 4), an assignment that seems highly unlikely given the unfavored status of the $v^{2}\left(8_{1}^{-}\right)$component as discussed above. In the case of ${ }^{252} \mathrm{No}$ $(N=150)$, our calculations predict that the $v^{2}\left(7 / 2^{+}[624]\right.$, 


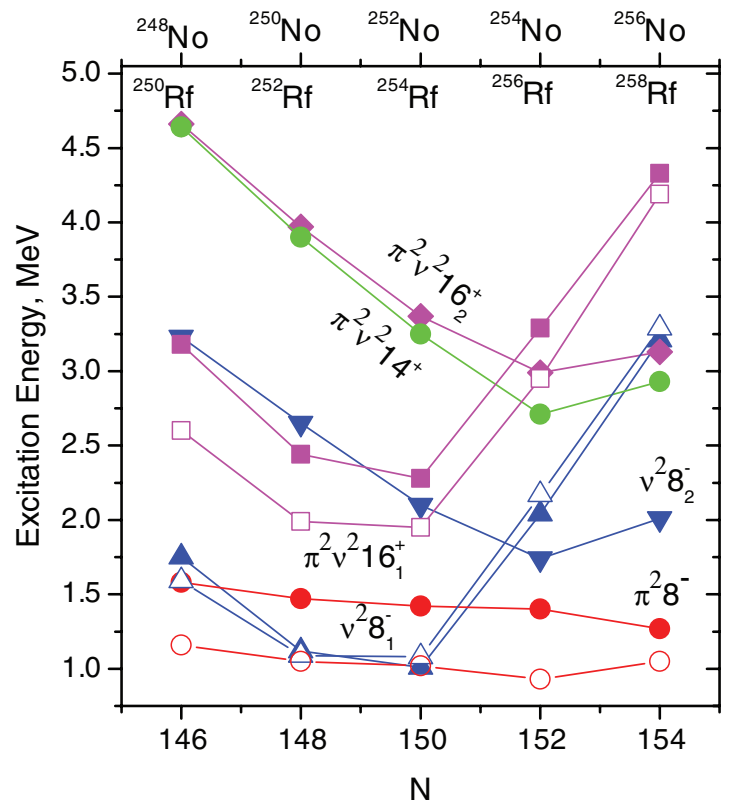

Fig. 4. Predicted two- and four-quasiparticle states in No (filled symbols) and $\mathrm{Rf}$ (open symbols) nuclei. Configurations: $\pi^{2} 8^{-}: \pi^{2}\left(7 / 2^{-}[514], 9 / 2^{+}[624]\right) ; v^{2} 8_{1}^{-}: v^{2}\left(7 / 2^{+}[624], 9 / 2^{-}[734]\right) ; v^{2} 8_{2}^{-}$: $v^{2}\left(7 / 2^{+}[613], 9 / 2^{-}[734]\right) ; \pi^{2} v^{2} 14^{+}: \pi^{2}\left(7 / 2^{-}[514], 9 / 2^{+}[624]\right) \otimes v^{2}\left(3 / 2^{+}\right.$ [622], 9/2- [734]); $\pi^{2} v^{2} 16_{1}^{+}: \pi^{2}\left(7 / 2^{-}[514], 9 / 2^{+}[624]\right) \otimes v^{2}\left(7 / 2^{+}[624]\right.$, $\left.9 / 2^{-}[734]\right) ; \pi^{2} v^{2} 16_{2}^{+}: \pi^{2}\left(7 / 2^{-}[514], 9 / 2^{+}[624]\right) \otimes v^{2}\left(7 / 2^{+}[613], 9 / 2^{-}\right.$ [734]).

$\left.9 / 2^{-}[734]\right)_{8_{1}^{-}}$configuration is lowest (at $1.01 \mathrm{MeV}$ ) and this has been confirmed by the recent observations of Robinson et al. [31]. It is worth noting that a very favorable $\mathrm{K}^{\pi}=16^{+}$ state formed by coupling of the $\pi^{2}\left(8^{-}\right)$and $v^{2}\left(8_{1}^{-}\right)$components is expected at $2.28 \mathrm{MeV}$ in ${ }^{252} \mathrm{No}$. It can decay via $\Delta \mathrm{K}=$ 8 forbidden transitions to members of the $\pi^{2}\left(8^{-}\right)$and/or $v^{2}\left(8_{1}^{-}\right)$ bands, and, hence, one may expect a long lifetime to be present. This state seems to be analogous to the $\mathrm{K}^{\pi}=16^{+}$ $\left(\mathrm{T}_{1 / 2}=31\right.$ (1) y) isomer in ${ }^{178} \mathrm{Hf}$ [32].

Calculations for $\operatorname{Rf}(Z=104)$ nuclei follow closely those for the No isotopes. For example, in ${ }^{256} \mathrm{Rf}(N=152)$, the $\pi^{2}\left(8^{-}\right)$configuration is predicted to be lowest and well separated from the competing $v^{2}\left(8^{-}\right)$ones. In ${ }^{254} \operatorname{Rf}(N=150)$, there is an apparent competition between the $\pi^{2}\left(8^{-}\right)$and $v^{2}\left(8_{1}^{-}\right)$ configurations. Although the calculations predict that the former would be favored, such a placement should be considered as tentative given the uncertainties in the calculation procedure (of order of $\sim 200 \mathrm{keV}$ ). Similar to ${ }^{252} \mathrm{No}$, a particularly favored $\mathrm{K}^{\pi}=16^{+}$, 4-quasiparticle state is predicted at $1.95 \mathrm{MeV}$, and one may expect an even longer-lived isomer in ${ }^{254} \mathrm{Rf}$. Given the fact that the ${ }^{254} \mathrm{Rf}$ ground state disintegrates by spontaneous fission $(\sim 100 \%)$, a search for this 4-quasiparticle state is particularly noteworthy since it can provide direct evidence for the role $\mathrm{K}$-isomers may play in the enhanced stability of super-heavy nuclei [28]. Because of blocking, the pairing correlations are reduced in the $\mathrm{K}^{\pi}=16^{+}$state, and, therefore, one may expect that the spontaneous-fission lifetimes increase considerably [21], as observed recently in ${ }^{250}$ No [33].
Future experimental work aimed at establishing rotational structures associated with the $\mathrm{K}^{\pi}=8^{-}$isomers in ${ }^{252,254} \mathrm{No}[23$, $24,31]$ would be valuable in order to elucidate the proposed configurations. More complete spectroscopic information is clearly needed in order to remove ambiguities in the decay of the 4-quasiparticle isomer in ${ }^{254}$ No.

This work is supported by the US Department of Energy, Office of Nuclear Physics, under Contracts No. DE-AC02-06CH11357 and DE-FG02-94ER40848, the ANSTO program for Access to Major Research Facilities, Grant No. 02/03-H-05 and the Australian Research Council Discovery projects DP0343027 and DP0345844.

\section{References}

1. R. Broda et al., Phys. Lett. B 251, 245 (1990).

2. R. Broda et al., Phys. Rev. C 49, 575(R) (1995).

3. R.D'Alarcao et al., Phys. Rev. C 59, R1227 (1999).

4. G.D. Dracoulis et al., Phys. Lett. B 584,22 (2004).

5. F.G. Kondev et al., Eur. Phys. J. A 22, 23 (2004).

6. G.D. Dracoulis et al., Phys. Rev. C 71, 044326 (2005).

7. G.D. Dracoulis et al., Phys. Lett. B 635, 200 (2006).

8. G.D. Dracoulis et al., Phys. Rev. Lett. 97, 122501 (2006).

9. E. Browne, H. Junde, Nucl. Data Sheets 87, 15 (1999).

10. F.G. Kondev et al. (to be published).

11. T.R. McGoram, G.D. Dracoulis, T. Kibédi, A.P. Byrne, R.A. Bark, A.M. Baxter, S.M. Mullins, Phys. Rev. C 62, 031303(R) (2000).

12. F.G. Kondev, M.A. Riley, D.J. Hartley, R.W. Laird, T.B. Brown, M. Lively, K.W. Kemper, J. Pfohl, S.L. Tabor, R.K. Sheline, Phys. Rev. C 59, R575 (1999).

13. F.G. Kondev et al., Phys. Lett. B 437, 35 (1998).

14. G.B. Hagemann et al., Nucl. Phys. A 618, 199 (1997).

15. P. Moller, J.R. Nix, W.D. Myers, W.J. Swiatecki, At. Data Nucl. Data Tables 59, 185 (1995).

16. P. Reiter et al., Phys. Rev. Lett. 82, 509 (1999).

17. M. Leino et al., Eur. Phys. J. A 6, 1 (1999).

18. R.-D. Herzberg et al., Phys. Rev. C 65, 014303 (2001).

19. J.E. Bastin et al., Phys. Rev. C 73, 024308 (2006).

20. R.R. Chasman, I. Ahmad, A.M. Friedman, J.R. Erskine, Rev. Mod. Phys. 49, 833 (1977).

21. S. Bjornholm, J.E. Lynn, Rev. Mod. Phys. 52, 725 (1980).

22. A. Ghiorso, K. Eskola, P., Eskola, M. Nurmia, Phys. Rev. C 7, 2032 (1973).

23. S.K. Tandel et al., Phys. Rev. Lett. 97, 082502 (2006).

24. R.-D. Herzberg et al., Nature 442, 896 (2006).

25. S. Cwiok, J. Dudek, W. Nazarewicz, J. Skalski, T. Werner, Comput. Phys. Commun. 46, 379 (1987).

26. W. Nazarewicz, M.A. Riley, J.D. Gareth, Nucl. Phys. A 512, 61 (1990).

27. F.G. Kondev, G.D. Dracoulis, A.P. Byrne, T. Kibédi, Nucl. Phys. A 632, 473 (1998).

28. F.R. Xu, E.G. Zhao, R. Wyss, P.M. Walker, Phys. Rev. Lett. 92, 252501 (2004).

29. V.G. Soloviev, Sov. J. Nucl. Phys. 54, 1232 (1991).

30. F.P. Heßberger et al., Eur. Phys. J. A 29, 165 (2006).

31. A. Robinson et al. (to be published).

32. R.G. Helmer, C.W. Reich, Nucl. Phys. A 114, 649 (1968).

33. D. Peterson et al., Phys. Rev. C 74, 014316 (2006). 